\title{
Qualidade de vida e satisfacão com o trabalho de profissionais das academias de ginástica da cidade de Pelotas/RS
}

\section{Quality of life and work satisfaction among fitness centers' instructors from Pelotas (Brazil)}

\author{
Lidiane Amanda Bevilacqua' \\ Marcelo Cozzensa da Silva ${ }^{1,2}$ \\ Felipe Fossati Reichert ${ }^{1,2}$ \\ Airton José Rombaldi ${ }^{1,2}$
}

\section{RESUMO}

A rotina de trabalho dos profissionais das academias de ginástica pode influenciar a sua percepção da qualidade de vida e satisfação no ambiente de trabalho. O presente estudo objetivou descrever a qualidade de vida e satisfação no trabalho de profissionais de academias de ginástica de Pelotas, RS. Foi conduzido um estudo tipo censo, transversal, com 497 profissionais. O Whoqol-Bref foi utilizado para mensuração da qualidade de vida e a satisfação profissional foi avaliada através do Occupational Stress Indicator. Dados sociodemográficos foram obtidos através de um questionário padronizado. $\mathrm{O}$ domínio meio ambiente apresentou o menor escore médio $(70,8)$ e o domínio relações sociais o maior escore $(82,2)$ de qualidade de vida. Os aspectos psicossociais com maior satisfação foram o relacionamento com outras pessoas no trabalho, conteúdo e motivação pela atividade realizada. O salário em relação ao tempo de experiência no trabalho, instabilidade no emprego e a falta de participação em decisões importantes foram apontados como maior insatisfação profissional. Os profissionais de academias apresentaram escores de qualidade de vida próximo ao máximo possível de ser atingido e estavam satisfeitos com seu trabalho. No entanto, precisa-se alterar as relações trabalhistas de modo a atender a legislação vigente.

\section{PALAVRAS-CHAVE}

Qualidade de vida; Satisfação no trabalho; Academias de ginástica.

\begin{abstract}
The routine work of fitness centers' instructors may influence their perception of quality of life and satisfaction in the workplace. This study aimed to describe the quality of life and work satisfaction among fitness centers' instructors from Pelotas, southern Brazil. We conducted a cross-sectional census-based study, with 497 professionals. The WHOQOL-Bref was used to measure quality of life and the Occupational Stress Indicator was used to assess work satisfaction. Sociodemographic data were also obtained. The environmental domain of quality of life presented the lowest mean score (70.8) and the social relationships domain the highest score (82.2). Higher satisfaction scores were obtained in the following psychosocial aspects: relationships with others at work, content and motivation for the activity performed. Salary, job instability and lack of participation in important decisions were associated with higher job dissatisfaction. Health clubs professionals presented scores close to the maximum score reached for quality of life and were satisfied with their work. However; relationships between employers and employees must advance with respect to labor laws.
\end{abstract}

\section{KEYWORDS}

Quality of life; Work satisfaction; Fitness Centers.
Rev Bras Ativ Fis Saúde p. 314-324 DOI

http://dx.doi.org/10.12820/rbafs.v.19n3p314

1 Curso de Mestrado em Educação Física Universidade Federal de Pelotas

2 GEEAF - Grupo de Estudos em Epidemiologia da Atividade Física - Universidade Federal de Pelotas 


\section{INTRODUÇÃ̃O}

As chamadas academias de ginástica, atuando como centros voltados à prática de exercícios físicos, sofreram inúmeras transformações ao longo do tempo ${ }^{1}$, de modo a adaptar-se as exigências do público frequentador. Assim, com a consolidação das academias de ginástica como espaço de cultura corporal, cresceu a demanda de profissionais especializados para atuar nesse âmbito de trabalho².

A rotina de trabalho desses profissionais possui como características uma ampla carga horária de trabalho e elevado esforço físico em condições nem sempre adequadas ${ }^{3}$, associados a baixas remunerações e incertezas geradas, muitas vezes, pela informalidade do trabalho ${ }^{4}$. Essas características podem interferir na percepção da qualidade de vida profissional e satisfação no ambiente de trabalho.

A qualidade de vida tem sido interesse de estudo em diferentes populações ${ }^{5}$. Estudos com profissionais ligados a área da saúde ${ }^{6}$, indivíduos com diferentes condições clínicas $^{7}$, adultos ${ }^{8}$ e idosos ${ }^{9}$ mediram qualidade de vida por diferentes instrumentos. A maior parte das pesquisas existentes investigou os professores de Educação Física no contexto escolar. Com esta população, os estudos indicaram melhores escores médios de qualidade de vida no domínio relações sociais, enquanto escores menores foram observados no domínio meio ambiente ${ }^{10-12}$. A rede de ensino (estadual), maior tempo de magistério e maior carga horária de trabalho semanal foram variáveis associadas a baixos escores na avaliação da qualidade de vida ${ }^{10}$. Estudos sobre a qualidade de vida de professores de educação física que trabalham com ginásticas, portanto, ainda são restritos.

Por outro lado, o ambiente de trabalho é um espaço que sofre constantes mudanças ${ }^{13}$. Nesse sentido, a satisfação profissional é um aspecto que pode influenciar no nível de qualidade de vida percebida, apresentando diferentes formas, dependendo do trabalho que desenvolve ${ }^{14}$.

Um estudo com enfermeiros conduzido no Brasil ${ }^{15}$ revelou que o relacionamento com outras pessoas, conteúdo do trabalho no qual desempenha, grau de motivação, grau de segurança no emprego e a flexibilidade e liberdade no local de trabalho são aspectos significativos associados a satisfação no trabalho.

Tendo em vista e escassez de relatos de pesquisa de base populacional realizados no Brasil abordando o tema nesse importante segmento de trabalhadores da área da saúde, o objetivo do presente estudo foi descrever a qualidade de vida e satisfação no trabalho dos profissionais de academias de ginástica de Pelotas, RS, Brasil.

\section{METODOLOGIA}

Foi realizado um estudo descritivo com delineamento transversal sobre a satisfação profissional e a qualidade de vida dos profissionais das academias de ginástica (graduados em educação física, não graduados, provisionados e estagiários) da cidade de Pelotas/RS.

Inicialmente dividiu-se a cidade de Pelotas em 19 setores, observando um espaço geográfico semelhante entre os mesmos. A fim de localizar todas as academias de ginástica existentes na cidade e obter informações básicas dos estabelecimentos e dos profissionais atuantes, direcionou-se entrevistadores treinados para o mapeamento. 
No total, 170 academias e 546 profissionais foram identificadas durante a coleta de dados que foi realizada no período de novembro de 2011 a maio de 2012. As informações foram obtidas por meio de entrevistadores universitários que passaram por um treinamento teórico-prático de 20 horas. Para garantir a padronização na coleta, também foi confeccionado um manual de instruções básicas para orientação.

Foram consideradas perdas os profissionais que não foram localizados por contato presencial e/ou telefônico e recusas os que expressaram verbalmente o desejo de não participar do estudo por mais de uma vez seguida, com intervalo mínimo de uma semana.

Foi utilizado o instrumento Word Health Organization Quality of Life Instrument Bref (WHOQOL-Bref) proposto pela Organização Mundial da Saúde, para avaliar a qualidade de vida ${ }^{16}$. O questionário é uma versão reduzida do Word Health Organization Quality of Life Instrument 100 (WHOQOL-100), composto de 26 questões, as quais medem a qualidade de vida em quatro domínios: físico, psicológico, relações sociais e meio ambiente. $\mathrm{O}$ domínio físico avalia dor e desconforto, energia e fadiga, sono e repouso; o domínio psicológico, os sentimentos positivos, pensar, aprender, memória e concentração, autoestima, imagem corporal (aparência), sentimentos negativos; o domínio relações sociais questiona as relações pessoais, suporte (apoio) social e atividade sexual; enquanto o domínio meio ambiente, a segurança física e proteção, ambiente no lar, recursos financeiros, cuidados de saúde e sociais, oportunidade de adquirir informações e habilidades, oportunidades de recreação e lazer, ambiente físico e locomoção. $\mathrm{O}$ instrumento gera escores que podem variar de zero a 100. Quanto mais elevado o escore obtido, melhor a qualidade de vida.

A satisfação profissional foi analisada através da escala de Satisfação no Trabalho do Occupational Stress Indicator (OSI) traduzido e validado para o português por Swan et al. ${ }^{17}$. Essa escala possibilita a avaliação dos sentimentos em relação a diferentes aspectos de trabalho. A mensuração da satisfação é obtida em 22 aspectos psicossociais no trabalho (salário, decisões, segurança, supervisão, potencialidades, mudança, conflitos, aspirações, carreira, comunicação, flexibilidade, volume, avaliação, crescimento, estrutura, clima, tarefa, desenvolvimento, imagem, relacionamento, conteúdo, motivação) por meio de escalas likert de seis pontos, classificados em: (1) enorme insatisfação, (2) muita insatisfação, (3) alguma insatisfação, (4) alguma satisfação, (5) muita satisfação e (6) enorme satisfação. A soma dessas medidas fornece um indicador de satisfação global no trabalho dado por um escore que varia de 22 a 132 pontos. No presente estudo, o escore da variável satisfação profissional foi utilizado de forma contínua e também categorizado. Tendo em vista a inexistência de um ponto de corte definido, o escore foi categorizado em três níveis: insatisfação (enorme insatisfação e muita insatisfação); satisfação intermediária (alguma insatisfação e alguma satisfação); e satisfação (muita satisfação e enorme satisfação) $^{18}$.

Adicionalmente, foram obtidas informações relacionadas as variáveis sexo, idade, estado civil, cor da pele, escolaridade, renda proveniente da academia, modalidades de aulas ministradas pelos professores, massa corporal e estatura autorreferidos e as variáveis comportamentais tabagismo, ingestão de álcool e atividade física. A variável idade foi coletada de forma contínua e poste- 
riormente categorizada em quatro níveis de faixa etária (17-29 anos; 30-39 anos; 40-49 anos; $\geq 50$ anos). A informação da renda foi obtida através da pergunta: "Qual é a sua renda mensal total bruta referente à(s) academia(s) que trabalha?". O estado nutricional foi verificado por meio do índice de massa corporal (IMC - calculado através das medidas da massa corporal e estatura autorreferidas) e os indivíduos classificados como sendo normais, com sobrepeso ou obesos ${ }^{19}$.

O critério utilizado para classificar o tabagismo foi aquele definido pela World Health Organization ${ }^{20}$, considerando fumante o indivíduo que fumou um ou mais cigarros por dia há pelo menos um mês. A variável consumo abusivo de álcool foi determinada segundo o critério do estudo Vigilância de Fatores de Risco e Proteção para Doenças Crônicas por Inquérito Telefônico ${ }^{21}$, do Ministério da Saúde do Brasil, o qual considera como abusivo o consumo de mais de cinco doses de bebida alcoólica para homens e mais de quatro doses para mulheres em uma mesma ocasião nos últimos 30 dias. O nível de atividade física nos domínios do lazer e do trabalho foram estimados pelo International Physical Activity Questionnaire (Questionário Internacional de Atividades Físicas) - IPAQ - versão longa ${ }^{22}$. Os indivíduos que relataram a prática semanal de atividade física nos domínios, igual ou superior a 150 minutos foram considerados ativos. Para construção deste escore, o tempo semanal com a prática de caminhada, atividades físicas moderadas e vigorosas foram somados, sendo que o tempo das atividades vigorosas foi multiplicado por dois ${ }^{23}$.

O estudo foi aprovado pelo Comitê de Ética em Pesquisa da Escola Superior de Educação Física da Universidade Federal de Pelotas-RS (nº 021/2011), e acompanha as Diretrizes e Normas Regulamentadoras de Pesquisas envolvendo Seres Humanos (Resolução n ${ }^{\circ}$ 196, de 10 de outubro de 1996) do Conselho Nacional de Saúde. Todos os entrevistados foram orientados sobre os objetivos do estudo e assinaram um termo de consentimento livre e esclarecido aceitando participar voluntariamente da pesquisa.

O banco de dados foi construído no Epidata 3.1, sendo realizada dupla digitação de cada questionário a fim de verificar erros de digitação. Para análise dos dados utilizou-se o programa Stata versão 10.0. Realizou-se uma análise descritiva para verificar médias e desvios padrão (DP) para as variáveis contínuas. Para as variáveis categóricas utilizou-se distribuição de frequências absolutas e relativas.

\section{RESULTADOS}

O total de 546 profissionais estava atuando nas academias de ginástica da zona urbana da cidade de Pelotas/RS no período da coleta dos dados. Desses, 497 profissionais participaram do estudo (58\% do sexo masculino), representando um percentual de perdas e recusas de $9,0 \%$. As variáveis IMC e renda foram as que apresentaram maior quantidade de valores ignorados (3 e 12, respectivamente). A média de idade encontrada foi de 29,7 anos (DP 8,4 anos), característico de uma população jovem (17 a 29 anos), recém-formada (52,3\% tinham até cinco anos de formados) e com pouco tempo de atuação em academias (51,3\% com no máximo 60 meses de experiência na área).

$\mathrm{Na}$ Tabela 1 verifica-se que a maioria dos indivíduos tinha entre 17 e 29 anos $(61,0 \%)$, era solteira $(66,4 \%)$, de cor de pele branca $(87,1 \%)$, com ní- 
vel superior completo $(65,4 \%)$ e que recebia até $\mathrm{R} \$ 1.000,00$ reais mensais $(52,4 \%)$ [o salário mínimo nacional era de $\mathrm{R} \$ 545,00$ no início da coleta de dados]. As modalidades mais ministradas nas academias de ginástica pelos professores foram musculação $(48,5 \%)$ e personal trainer $(38,8 \%)$.

TABELA 1 - Características dos profissionais de academias de ginástica, de acordo com o sexo, da cidade de Pelotas/RS (n=497), 2012.

\begin{tabular}{|c|c|c|c|c|}
\hline \multirow{2}{*}{ Variáveis } & \multicolumn{2}{|c|}{ Masculino } & \multicolumn{2}{|c|}{ Total } \\
\hline & $\mathrm{N}$ & $\%$ & $\mathrm{n}$ & $\%$ \\
\hline \multicolumn{5}{|l|}{ Idade } \\
\hline 17-29 anos & 162 & 56,3 & 303 & 61,0 \\
\hline 30-39 anos & 93 & 32,3 & 139 & 28,0 \\
\hline 40-49 anos & 22 & 7,6 & 36 & 7,2 \\
\hline$\geqslant 50$ anos & 11 & 3,8 & 19 & 3,8 \\
\hline \multicolumn{5}{|l|}{ Estado civil } \\
\hline Casado (a) ou vive com companheiro (a) & 91 & 31,6 & 146 & 29,4 \\
\hline Solteiro (a) & 187 & 64,9 & 330 & 66,4 \\
\hline Separado (a) & 10 & 3,5 & 21 & 4,2 \\
\hline \multicolumn{5}{|l|}{ Cor da pele } \\
\hline Branca & 240 & 83,3 & 433 & 87,1 \\
\hline Parda /preta & 48 & 16,7 & 64 & 12,9 \\
\hline \multicolumn{5}{|l|}{ Escolaridade } \\
\hline Até Fundamental completo & 7 & 2,4 & 9 & 1,8 \\
\hline Médio incompleto /completo & 42 & 12,5 & 42 & 8,5 \\
\hline Superior incompleto & 121 & 26,4 & 121 & 24,3 \\
\hline Superior completo & 325 & 58,7 & 325 & 65,4 \\
\hline \multicolumn{5}{|l|}{ Renda proveniente da academia } \\
\hline Até $1.000,00$ reais & 140 & 50,0 & 254 & 52,4 \\
\hline De $1.001,00$ à $2.500,00$ reais & 101 & 36,1 & 160 & 33,0 \\
\hline De $2.501,00$ à $5.000,00$ reais & 25 & 8,9 & 51 & 10,5 \\
\hline Acima de $5.001,00$ reais & 14 & 5,0 & 20 & 4,1 \\
\hline \multicolumn{5}{|l|}{ Modalidades ministradas* } \\
\hline Musculação & 153 & 53,1 & 241 & 48,5 \\
\hline Personal Trainer & 114 & 39,6 & 193 & 38,8 \\
\hline Ginásticas & 28 & 9,7 & 83 & 16,7 \\
\hline Lutas & 71 & 24,7 & 76 & 15,3 \\
\hline Pilates & 22 & 7,6 & 73 & 14,7 \\
\hline Dança & 13 & 4,5 & 33 & 6,6 \\
\hline Atividades aquáticas & 11 & 4,2 & 31 & 6,2 \\
\hline Yoga & 2 & 0,7 & 9 & 1,8 \\
\hline \multicolumn{5}{|l|}{ Tabagismo } \\
\hline Nunca fumou & 221 & 76,8 & 368 & 74,1 \\
\hline Ex-fumante & 62 & 21,5 & 117 & 23,5 \\
\hline Fumante atual & 5 & 1,7 & 12 & 2,4 \\
\hline \multicolumn{5}{|l|}{ Ingestão de álcool } \\
\hline Acima recomendado & 111 & 55,7 & 359 & 55,4 \\
\hline Recomendado** & 88 & 44,3 & 160 & 44,6 \\
\hline \multicolumn{5}{|l|}{ Índice de massa corporal } \\
\hline Normal & 106 & 37,1 & 382 & 57,1 \\
\hline Sobrepeso & 152 & 53,1 & 177 & 35,8 \\
\hline Obeso & 28 & 9,8 & 35 & 7,1 \\
\hline \multicolumn{5}{|l|}{ Atividade Física no trabalho } \\
\hline Insuficientemente ativo & 75 & 26,0 & 123 & 24,7 \\
\hline Ativo & 213 & 74,0 & 374 & 75,3 \\
\hline \multicolumn{5}{|l|}{ Atividade Física no Lazer } \\
\hline Insuficientemente ativo & 46 & 16,0 & 103 & 20,7 \\
\hline Ativo & 242 & 84,0 & 394 & 79,3 \\
\hline
\end{tabular}

* Maior que $100 \%$, pois um profissional pode atuar em mais de uma modalidade.

$* * \leqslant 5$ doses para homens numa mesma ocasião e $\leqslant 4$ doses para mulheres numa mesma ocasião 
A maioria $(74,1 \%)$ relatou nunca ter fumado na vida e pouco mais da metade dos entrevistados apresentou consumo consumo abusivo de álcool $(55,4 \%)$. Em relação ao IMC, 53,1\% dos homens foi classificado com sobrepeso, enquanto a maioria das mulheres $(84,1 \%)$ classificou-se com peso normal. Além disso, a maioria era ativo em relação às atividades desenvolvidas no trabalho $(75,3 \%)$ e no domínio do lazer $(79,3 \%)$.

A média dos escores obtidos em cada domínio da qualidade de vida é apresentada na Tabela 2 . Além de escores elevados de qualidade de vida, em ambos os sexos o domínio meio ambiente apontou as menores médias $(70,8)$ e o domínio social os valores mais elevados $(82,2)$.

TABELA 2 - Médias (desvios-padrão) dos escores dos domínios de qualidade de vida (WHOQOL -bref) de profissionais de academias de ginástica. Pelotas/RS (n=497), 2012.

\begin{tabular}{lccccc}
\hline \multirow{2}{*}{$\begin{array}{l}\text { Domínio da Qualidade de } \\
\text { Vida (WHOQOL-bref) }\end{array}$} & \multicolumn{2}{c}{ Masculino } & \multicolumn{2}{c}{ Feminino } & Total \\
\cline { 2 - 5 } & Média (DP) & Min - Max & Média (DP) & Min - Max & Média (DP) \\
\hline Físico & $80,3(12,3)$ & $14,3-100$ & $77,0(13,2)$ & $25-100$ & $78,9(12,8)$ \\
\hline Social & $82,9(14,2)$ & $16,6-100$ & $81,3(14,7)$ & $25-100$ & $82,2(14,5)$ \\
\hline Psicológico & $80,2(11,1)$ & $33,4-100$ & $75,8(11,2)$ & $45,8-100$ & $78,4(11,4)$ \\
\hline Meio Ambiente & $71,3(12,4)$ & $34,4-100$ & $70,1(13,2)$ & $31,3-96,9$ & $70,8(12,7)$ \\
\hline
\end{tabular}

DP = desvio padrão; Min = valor mínimo encontrado. Max= valor máximo encontrado

A frequência relativa encontrada nos aspectos psicossociais da escala de satisfação no trabalho (OSI) é descrita na Tabela 3. Observou-se que os profissionais estavam bastante satisfeitos na maioria dos aspectos do trabalho. Os aspectos com maior proporção de profissionais satisfeitos foram o relacionamento com outras pessoas (94,7\%), conteúdo do trabalho que realiza $(86,2 \%)$ e grau de motivação para o trabalho (85,7\%). Já os aspectos com maior proporção de profissionais insatisfeitos foram o salário em relação à experiência e à responsabilidade que tem, o grau de participação em decisões importantes e o grau de segurança (falta de estabilidade) no emprego (13,5\%, 9,2\% e 7,4\% respectivamente).

A Figura 1 apresenta a variável satisfação profissional categorizada em três níveis (satisfeitos, intermediário e insatisfeitos) de acordo com o sexo. Observou-se que a maioria dos entrevistados $(88,9 \%)$ apresentou percentuais de satisfação no ambiente de trabalho próximo do máximo valor possível de ser obtido (90,3\% e $87,1 \%$ para homens e mulheres, respectivamente).

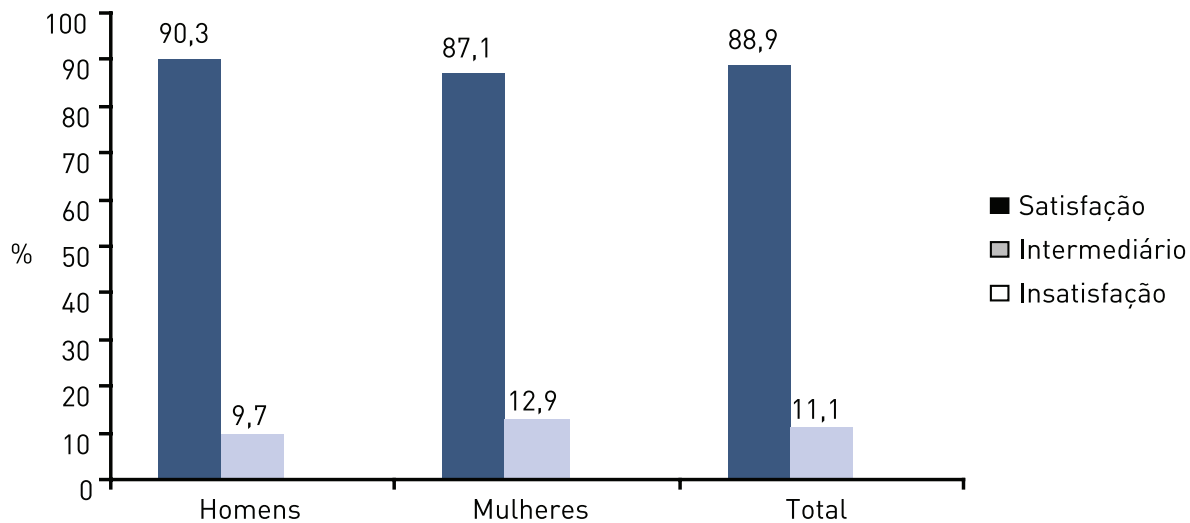

FIGURA 1 - Nível de satisfação no trabalho de profissionais de academias de ginástica de Pelotas/ RS, segundo o sexo. RS (n=497), 2012. 
TABELA 3 - Aspectos psicossociais e satisfação no trabalho de profissionais de academias de ginástica de Pelotas/RS (n=497), 2012.

\begin{tabular}{|c|c|c|c|}
\hline & Insatisfação & Intermediário & Satisfação \\
\hline & \multicolumn{3}{|c|}{$\%$} \\
\hline Salário & 13,4 & 44,9 & 41,7 \\
\hline Decisões & 9,2 & 28,4 & 62,4 \\
\hline Segurança & 7,4 & 32,2 & 60,4 \\
\hline Supervisão & 6,9 & 29,7 & 63,4 \\
\hline Potencialidades & 3,6 & 20,6 & 75,8 \\
\hline Mudança & 5,8 & 34,4 & 59,8 \\
\hline Conflitos & 6 & 39,4 & 54,6 \\
\hline Aspirações & 3,2 & 31 & 65,8 \\
\hline Carreira & 3,4 & 34,4 & 62,2 \\
\hline Comunicação & 2,4 & 29,4 & 68,2 \\
\hline Flexibilidade & 2,4 & 13,1 & 84,5 \\
\hline Volume & 2,8 & 36,3 & 60,9 \\
\hline Avaliação & 2 & 25,3 & 72,7 \\
\hline Crescimento & 2 & 17,7 & 80,3 \\
\hline Estrutura & 2,4 & 36,7 & 60,9 \\
\hline Clima & 1,6 & 15,3 & 83,1 \\
\hline Tarefa & 1 & 27,7 & 71,3 \\
\hline Desenvolvimento & 1,8 & 23,5 & 74,7 \\
\hline Imagem & 1,2 & 24,4 & 74,4 \\
\hline Relacionamento & 0,2 & 5 & 94,8 \\
\hline Conteúdo & 0,6 & 13,3 & 86,1 \\
\hline Motivação & 0,2 & 14,1 & 85,7 \\
\hline
\end{tabular}

\section{DISCUSSÃO}

Foram localizados poucos estudos com profissionais de educação física atuando profissionalmente em academias de ginástica. A maior parte dos estudos que investigaram a qualidade de vida de professores está relacionada ao contexto escolar ${ }^{24-25}$. Investigando a qualidade de vida de professores de escolas públicas em diferentes cidades do país ${ }^{11,12,26}$, foram encontrados resultados semelhantes com o do presente estudo, onde o escore médio mais alto da qualidade de vida foi encontrado no domínio social (82,2 pontos) e o mais baixo no domínio meio ambiente (70,8 pontos). Embora haja discrepâncias entre o contexto escolar e o ambiente das academias, percebe-se que ambos profissionais mantêm algumas características em comum. A proximidade com os alunos e forma de convívio com as pessoas, são características do profissional de Educação física.

Xavier e Morais ${ }^{12}$, em estudo com professores de escolas públicas estaduais da cidade de Aracajú/SE, corroboram o presente estudo ao relatarem em seus achados que o escore mais elevado da qualidade de vida foi no domínio social (70,2 pontos), enquanto o menor foi encontrado no domínio meio ambiente (51,6 pontos). Os autores atribuem o baixo escore a um ambiente de trabalho com condições desfavoráveis para a prática das aulas, tanto do ponto de vista térmico como acústico, além dos ambientes serem pequenos e inadequados. 
Penteado e Pereira ${ }^{11}$ analisando 128 professores de escolas públicas estaduais da cidade de Rio Claro/SP, e Silva e Nunes ${ }^{26}$ investigando professores de Educação Física da rede municipal de ensino de Campo Grande/MS encontraram escores médios inferiores ao do presente estudo nos domínios da qualidade de vida, principalmente no domínio físico. Tais diferenças podem ser atribuídas à maior idade média e ao maior tempo de serviço relatados nos estudos ${ }^{11,26}$ quando comparados com o presente estudo.

Outro fator determinante nas relações pessoais e que influencia na saúde e bem estar do indivíduo é a satisfação profissional. Além do estado emocional que pode indicar alegrias (satisfação) ou desconforto/desprazer (insatisfação), as consequências da falta de satisfação no trabalho pode interferir na saúde física e mental, qualidade de vida, estresse, autoestima e na vida individual ${ }^{27}$. Neste sentido, os profissionais de academias de ginástica da zona urbana da cidade de Pelotas/RS consideraram-se satisfeitos com os aspectos psicossociais no ambiente de trabalho. Corroborando resultados apresentados em outros estudos nacionais ${ }^{15,18}$, os aspectos com maior proporção de satisfação foram relacionados ao conteúdo do trabalho que realizam, relacionamento com outras pessoas e grau de motivação para o trabalho.

Professores de educação física da rede estadual do estado do Paraná consideraram como fatores de satisfação no exercício da docência, a progressão na carreira através de leis que garantiam seus direitos e deveres, proporcionando condições de desenvolverem suas capacidades e a percepção de sua importância social ${ }^{28}$. Esse fator de satisfação dificilmente é encontrado nos profissionais de academias de ginástica, pois poucos apresentam vínculo empregatício, não existindo, portanto, essa condição de progressão na carreira.

Os aspectos de maior insatisfação apontados pelos profissionais investigados estiveram relacionados ao salário que recebiam, ao grau de participação em decisões importantes e o grau de segurança (falta de estabilidade) no emprego, referendando resultados encontrados em outros estudos onde a insatisfação com o salário ${ }^{15,24,28}$ e segurança no emprego ${ }^{18}$ também foram ressaltados. Estudo com docentes universitários brasileiros ${ }^{18}$ corrobora os resultados do presente estudo ao reportarem que a categoria mais prevalente de respostas em relação ao salário, foi de satisfação intermediária.

Diferentemente dos resultados encontrados, estudo em outras populações, como por exemplo, trabalhadores do sistema elétrico ${ }^{29}$, relataram que os principais aspectos psicossociais de insatisfação no ambiente de trabalho, diziam respeito a como as mudanças e inovações eram implementadas, o grau que a empresa se aproveitava do potencial que o trabalhador julgava ter e a maneira em que os conflitos eram resolvidos. Neste sentido, o tipo de trabalho desenvolvido pode ser determinante da dimensão atribuída à satisfação associada ao ambiente de trabalho.

Analisando o estresse ocupacional de bancários, Michailidis e Georgiou ${ }^{30}$ confirmaram que os funcionários que ocupam cargos mais altos são mais satisfeitos com seu trabalho. Pesquisas com trabalhadores das áreas da saúde e da educação salientam que cargos mais elevados de ocupação, a escolha do local de trabalho e o gênero contribuem significativamente para a satisfação profissional ${ }^{31,32}$.

Em relação às variáveis qualidade de vida e satisfação profissional, foram encontradas elevadas prevalências nos profissionais de educação física que atuam em academias de ginástica na cidade de Pelotas/RS. A literatura é ine- 
xistente nesse aspecto, impossibilitando comparações. Esse achado pode estar relacionado à associação desse profissional à hábitos saudáveis, como baixas prevalências de tabagismo e elevados níveis de atividade física, e também a baixa média de idade encontrada nesses trabalhadores. Outra possível explicação pode estar atrelada as características do profissional atuante em academias de ginástica, considerando que estes, na sua maioria são indivíduos jovens, sadios, com estética corporal condizente com os padrões de beleza para a sociedade, condições importantes para a imagem agregada ao espaço das academias.

Concluindo, foi observado que os profissionais de academias apresentaram escores de qualidade de vida, em todos os domínios, próximos do valor mais máximo possível de ser obtido, sendo o mais elevado no domínio social. Quanto aos aspectos psicossociais com maior proporção de profissionais no nível satisfação, concluiu-se que estes profissionais estavam satisfeitos no ambiente de trabalho principalmente com a atividade realizada, motivação e o relacionamento com as outras pessoas no trabalho. Os aspectos com maior proporção de insatisfação (salário, falta de estabilidade no emprego e participação em decisões importantes), corroboram com as características desse mercado de trabalho, muitas vezes informal, sem acordos trabalhistas e com constante substituição do quadro funcional.

Apesar dos significativos escores de qualidade de vida e satisfação com o trabalho verificados nos entrevistados, questões relacionadas a legislação trabalhista foram apontadas como causadoras de insatisfação. A categoria profissional de educação física precisa, nas instâncias apropriadas, discutir essas questões buscando alternativas concretas para a mudança do quadro. Adicionalmente, salienta-se a importância da realização de estudos com esses profissionais onde a exigência física é constantemente enfatizada refletindo na saúde geral do indivíduo e suas ações mentais.

\section{Colaboradores}

LAB participou de todas as etapas da pesquisa e redação do artigo. AJR, MCS e FFR planejaram o estudo, coordenaram sua execução e colaboraram na análise dos dados, escrita e revisão final do artigo.

\section{REFERÊNCIAS}

1. Furtado RP. Do fitness ao wellness: os três estágios de desenvolvimento das academias de ginástica. Pensar Prat 2009; 12:1-11.

2. Coelho Filho CAA, Frazão DP. Prática de ginástica em academias exclusivamente femininas. Motriz 2010; 16:269-80.

3. Palma A, Azevedo APG, Ribeiro SSM, Santos TF, Nogueira L. Saúde e trabalho dos professores de educação física que atuam com atividades aquáticas. Arq Mov 2006; 2:81-101.

4. Palma A. Vida de professores de educação física que atuam em academias de ginástica: comportamento de risco ou vulnerabilidade? Anais da II Conferência do Imaginário e das Representações Sociais em Educação Física, Esporte e Lazer. Rio de Janeiro: Universidade Gamo Filho, 2003, p. 883.

5. Pucci GCMF, Rech CR, Fermino RC, Reis RS. Association between physical activity and quality of life in adults. Rev Saúde Pública 2012; 46:166-179.

6. Silva AA, Souza JMP, Borges FNS, Fischer FM. Health-related quality of life and working conditions among nursing providers. Rev Saúde Pública 2010; 44: 718-25. 
7. Agarwal R, Goldenberg M, Perry R, Ishak WW. The quality of life of adults with attention deficit hyperactivity disorder: a systematic review. Innov Clin Neurosci 2012; 9:10-21

8. Anokye NK, Trueman P, Green C, Pavey TG, Taylor RS. Physical activity and health related quality of life. BMC Public Health 2012; 12:624.

9. Olivares PR, Gusi N, Prieto J, Mocholi MAH. Fitness and health-related quality of life dimensions in community-dwelling middle aged and older adults. Health Qual Life Outcomes 2011; 9:117.

10. Pereira EF, Teixeira CS, Lopes AS. Qualidade de vida de professores de educação básica do município de Florianópolis, SC, Brasil. Cien Saude Colet 2013; 18:1963-1970.

11. Penteado RZ, Pereira IMTB. Qualidade de vida e saúde vocal de professores. Rev Saúde Pública 2007; 41:236-43.

12. Xavier CES, Morais AS. Qualidade de vida em professores da rede pública estadual de ensino da cidade de Aracaju - SE. Rev Bras Presc Fisiol Exerc 2007; 1:85-94.

13. Siqueira VTA, Kurcgant P. Job Satisfaction: a quality indicator in nursing human resource management. Rev Esc Enferm 2012; 46:146-52.

14. Schmidt DRC, Dantas RAS. Qualidade de vida no trabalho de profissionais de enfermagem, atuantes em unidades do bloco cirúrgico, sob a ótica da satisfação. Rev Latino Am Enfermagem 2006; 14:54-60.

15. Chaves LD, Ramos LH, Figueiredo EN. Job satisfaction of nurses working in Brazil. Acta Paul Enferm 2011; 24:507-13.

16. The WHOQOL Group. Development of the world Health organization WHOQOLBref quality of life assessment. Psychol Med 2000; 28:551-558.

17. Swan JA, Moraes LFR, Cooper CL. Developing the occupational stress indicator (OSI) for use in Brazil: a report on the reliability and validity of the translated OSI. Stress Med 1993; 9:247-53.

18. Marqueze, EC, Moreno CRC. Satisfação no trabalho e capacidade para o trabalho entre docentes universitários. Psicol Estud 2009; 14:75-82.

19. World Health Organization (WHO). Physical status: the use and interpretation of anthropometry. Geneva, Technical Report Series 854, 1995.

20. World Health Organization (WHO). Definitions of smoking. Global link. Geneva; 2003.

21. Ministério da Saúde. VIGITEL Brasil 2010: vigilância de fatores de risco e proteção para doenças crônicas por inquérito telefônico. Brasília: Ministério da Saúde; 2010:152.

22. Craig CL, Marshall AL, Sjostrom M, Bauman AE, Booth ML, Ainsworth BE, et al. International physical activity questionnaire: 12 -coutry reability and validity. Med Sci Sports Exerc 2003; 35:1381-95.

23. U.S. Department Of Health And Human Services. Physical Activity Guidelines for Americans. Washington, DC: U.S. Department of Health and Human Services; 2008. Disponível em: http://www.health.gov/paguidelines, acesso 15 de julho de 2011.

24. Moreira HR, Nascimento JV, Sonoo CN, Both J. Qualidade de vida do trabalhador docente em Educação Física do estado do Paraná, Brasil. Rev Bras Cineantropom Desempenho Hum 2010; 12:435-42.

25. Folle A, Farias G. Nível de qualidade de vida e de atividade física de professores de escolas públicas estaduais da cidade de Palhoça (SC). Rev Mackenzie Educ Fís Esporte 2012; 11:11-21.

26. Silva JVP, Nunez PRM. Qualidade de vida, perfil demográfico e profissional de professores de educação física. Pensar Prat 2009; 12:1-11.

27. Faragher EB, Cass M, Cooper CL. The relationship between job satisfaction and health: a meta-analysis. Occup Environ Med 2005; 62:105-112.

28. Moreira HR, Nascimento JV, Sonoo CN, Both J. Qualidade de vida do trabalhador docente e os ciclos vitais de professores de Educação Física do Estado do Paraná, Brasil. Rev bras Cien Mov 2010; 18:12-20.

29. Macaia AAS, Marqueze EC, Rotenberg L, Fischer FM, Moreno CRC. Job satisfaction among control room operators of electrical systems. Work 2012; 41:5459-61. 
30. Michailidis M, Georgiou Y. Employee occupational stress in banking. Work 2005; 24:123-37.

31. Ward M, Cowman S. Job satisfaction in psychiatric nursing. J Psychiatr Ment Health Nurs 2007; 14:454-61.

32. Okpara JO, Squillace M, Erondu EA. Gender differences and job satisfaction: a study of university teachers in the United States. Women Manage Rev 2005; 20:177-190.

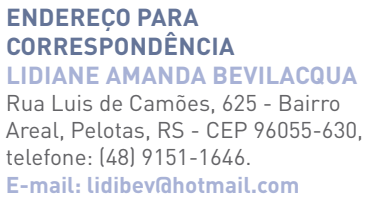

